\title{
EDITORIAL
}

nature

biotechnology

\section{Best-kept secrets}

\author{
Industry needs to fully embrace efforts by regulators to release clinical trial data on approved products, despite lingering \\ concerns over the public release of confidential business information. It is simply the right thing to do.
}

ater this month, the Management Board of the European Medicines LAgency (EMA) will meet to discuss the agency's finalized policy on the publication of, and access to, clinical trial data. As a response to this policy and similar transparency initiatives at the US Food and Drug Administration (FDA), industry trade associations have put forward their own principles for voluntarily making human testing data available to "qualified researchers." Although these moves to greater transparency are a positive development, they are being played out against a backdrop in which company secrecy-specifically confidential business information (CBI) - is on the rise. According to the Feature on p. 528, the use of $\mathrm{CBI}$ is increasing not only for drug products, but also for biotech seed products. As society as a whole moves toward greater openness, industry must be careful to balance its imperative to protect CBI with the calls from researchers, physicians and patients for greater transparency.

CBI is an umbrella term for what are known legally as 'trade secrets'. It refers to commercially valuable information that is not widely known to other businesses and thus gives its owner a competitive advantage. A trade secret can refer to any information (product composition/formulation, device, method, technique or process) that has value as long as it remains hidden from competitors. A classic example is the formula of Coca-Cola.

The biotech industry has traditionally used a mix of intellectual property (IP) strategies for its platforms and products, including the filing of patents, the publication of scientific papers and the use of trade secrets. As the industry has matured, however-and larger, more profitable biotech firms have come to the fore-the tendency towards trade secrets has increased. In other words, biotech companies are beginning to resemble their larger, more secretive, pharmaceutical brethren.

According to data gathered by Nature Biotechnology, plant biotech has witnessed a tenfold increase in the use of CBI since 1993. Indeed, CBI is invoked with increasing regularity, despite the fact that regulatory registrations have been on the decline since 2002. In the biopharmaceutical sector, increasing tracts of regulatory documents describing the constructs and sequences of biologic drugs are redacted as CBI, making it difficult to tell exactly the composition of newly approved products.

One reason why trade secrets are becoming so pervasive is that legal definitions of what is and what is not CBI are so vague. This gives companies leeway to invoke CBI in all manner of contexts, often with little justification. At the same time, regulators' default attitude is to redact any of the documentation associated with a product registration that could be construed as CBI because of the substantial fines and even jail time associated with disclosure of trade secrets.

Another explanation for the rise of CBI is the increasingly competitive business environment; today, manufacturers must contend not only with other brands seeking to take their market share, but also with the prospect of biosimilar competitors. In this context, it is very much in the interest of innovator companies to place off limits an increasing amount of information relating to clinical trial design and strategy, cell growth conditions, analytical, manufacturing and purification process, and even characteristics of producer cell lines.

The area where CBI has sparked most controversy is its use to block access to missing clinical data on approved products. This is a serious problem because physicians often lack the complete set of safety and efficacy data needed to understand how best to prescribe a drug. In 2007, for example, EMA cited CBI as the central justification for denying Cochrane Collaboration researchers access to clinical data on the diet drugs Xenical and Acomplia. The dispute subsequently ended up with the European Ombudsman, who ruled in 2010 that "clinical trial data do not contain commercially confidential information, and therefore EMA cannot refuse access." Ever since that case, political pressure has been growing to increase data transparency and address the important problem of missing trial data on marketed drugs.

Last June, EMA drew up its policy on releasing trial data, placing summary and aggregate clinical data about approved products online, allowing researchers access to patient-level data and encouraging applicants to submit a prespecified data analysis plan. The FDA also drafted a proposal to make patient-level data available, the main difference being the information identifies only the drug class rather than individual products.

Needless to say, the Pharmaceutical Research and Manufacturers of America (PhRMA) and the Biotechnology Industry Organization (BIO) have not welcomed these transparency initiatives with open arms. Instead, they would prefer that member companies voluntarily create their own access programs and control on an individual basis the types of information released and the people to whom that information is released.

The problem with this is that voluntary programs are not likely to engage enough companies to address the pernicious issue of missing clinical data. Some companies like GlaxoSmithKline and Roche may be prominent adopters of transparency, but how many of the other thousands of companies are likely to follow them without a business or regulatory incentive to do so?

More to the point, is the type of data that regulators are planning to release on approved products really so business critical that it can only be trusted in the hands of companies? The European Ombudsman, European Parliament and member states believe not. In which case, the key question is, why shouldn't the public and research community have the right to access all the relevant clinical trial data relating to the clinical safety and efficacy of products that have been approved for market release?

At the moment, no one in industry has provided an answer. And until someone does, all the foot-dragging and legal wrangling with regulators is going to reinforce one thing: the perception that this is an industry primarily driven by profit, rather than the needs of the patients it serves. 